\title{
Positive solutions for boundary value problems of fractional difference equations depending on parameters
}

Shugui Kang ${ }^{1 *}$, Xiaohong Zhao ${ }^{2}$ and Huiqin Chen ${ }^{1}$

\section{"Correspondence:}

dtkangshugui@126.com

${ }^{1}$ Applied of Mathematics Institute,

Shanxi Datong University, Datong,

Shanxi 037009, P.R. China

Full list of author information is

available at the end of the article

\begin{abstract}
We use the Krasnosel'skii fixed point theorem to obtain the sufficient conditions of the existence of two positive solutions for the boundary value problem of fractional difference equations depending on parameters.
\end{abstract}

MSC: 26A33; 39A05; 39A 12

Keywords: discrete boundary value problem; fixed point theory; cone; positive solution

\section{Introduction}

In this paper, we consider the boundary value problems of fractional difference equations depending on parameters of the form

$$
\begin{aligned}
& -\Delta^{v_{j}} y_{j}(t)=\lambda_{j} f_{j}\left(y_{1}\left(t+v_{1}-1\right), \ldots, y_{n}\left(t+v_{n}-1\right)\right), \\
& y_{j}\left(v_{j}-2\right)=\psi_{j}\left(y_{j}\right), \quad y_{j}\left(v_{j}+b\right)=\phi_{j}\left(y_{j}\right),
\end{aligned}
$$

where $t \in[0, b]_{\mathbb{N}_{0}}:=\{0,1, \ldots, b\}, \lambda_{j}>0,1<v_{j} \leq 2, f_{j}:[0,+\infty) \times \cdots \times[0,+\infty) \rightarrow[0,+\infty)$ are continuous functions. For each $j$, we have that $\psi_{j}, \phi_{j}: \mathbb{R}^{b+3} \rightarrow \mathbb{R}(j=1,2, \ldots, n)$ are given functions. We point out that fractional difference equations have been extensively studied in recent years. Systems of discrete fractional boundary value problems are also popular. In [1], the authors discussed the existence of positive solutions for coupled systems of nonlinear fractional difference equations:

$$
\begin{aligned}
& \Delta^{2} u(n-1)+\lambda a(n) f(u(n), v(n))=0, \\
& \Delta^{2} v(n-1)+\mu b(n) g(u(n), v(n))=0, \\
& u(0)=\beta u(\eta), \quad u(N)=\alpha u(\eta), \quad v(0)=\beta v(\eta), \quad v(N)=\alpha v(\eta),
\end{aligned}
$$

where $\eta \in\{1, \ldots, N-1\}, n \in\{1, \ldots, N-1\}, N \geq 4, \alpha, \beta, \lambda, \mu>0$. In [2], Goodrich studied the following pair of discrete fractional boundary value problems:

$$
\begin{aligned}
& -\Delta^{v_{1}} y_{1}(t)=\lambda_{1} a_{1}\left(t+v_{1}-1\right) f_{1}\left(y_{1}\left(t+v_{1}-1\right), y_{2}\left(t+v_{2}-1\right)\right), \\
& -\Delta^{v_{2}} y_{2}(t)=\lambda_{2} a_{2}\left(t+v_{2}-1\right) f_{2}\left(y_{1}\left(t+v_{1}-1\right), y_{2}\left(t+v_{2}-1\right)\right),
\end{aligned}
$$

○2013 Kang et al.; licensee Springer. This is an Open Access article distributed under the terms of the Creative Commons Attribution License (http://creativecommons.org/licenses/by/2.0), which permits unrestricted use, distribution, and reproduction in any medium, provided the original work is properly cited. 


$$
\begin{array}{ll}
y_{1}\left(v_{1}-2\right)=\psi_{1}\left(y_{1}\right), & y_{2}\left(v_{2}-2\right)=\psi_{2}\left(y_{2}\right), \\
y_{1}\left(v_{1}+b\right)=\phi_{1}\left(y_{1}\right), & y_{2}\left(v_{2}+b\right)=\phi_{2}\left(y_{2}\right),
\end{array}
$$

where $t \in[0, b]_{\mathbb{N}_{0}}:=\{0,1, \ldots, b\}, \lambda_{1}, \lambda_{2}>0, v_{1}, v_{2} \in(1,2]$, it is the same as [1] when $v_{1}, v_{2}=2$. Goodrich obtained the existence of at least one positive solution to this problem by means of the Krasnosel'skii theorem for cones. We shall deduce the existence of at least two positive solutions to problem (1.1)-(1.2) in this paper. These results extend the results of [2].

The paper is organized as follows. In Section 2, we present basic definitions and demonstrate some lemmas in order to prove our main results. In Section 3, we establish some results for the existence of at least two solutions to problem (1.1)-(1.2), and we present an example to illustrate our main results.

\section{Preliminaries}

For the convenience of the reader, we give some definitions and fundamental facts of the discrete fractional calculus, which can be found in [3-6] and their references.

Definition 2.1 [3] We define

$$
t^{(v)}=\frac{\Gamma(t+1)}{\Gamma(t+1-v)}
$$

for any $t$ and $v$, for which the right-hand side is defined. We also appeal to the convention that if $t+1-v$ is a pole of the gamma function and $t+1$ is not a pole, then $t^{(v)}=0$.

Definition 2.2 [3] The $v$ th fractional sum of a function $f$ defined on the set $\mathbb{N}_{a}:=\{a, a+$ $1, \ldots\}$, for $v>0$, is defined to be

$$
\Delta^{-v} f(t)=\Delta^{-v} f(t ; a):=\frac{1}{\Gamma(v)} \sum_{s=a}^{t-v}(t-s-1)^{(v-1)} f(s),
$$

where $t \in\{a+v, a+v+1, \ldots\}=: \mathbb{N}_{a+v}$. We also define the $v$ th fractional difference for $v>0$ by

$$
\Delta^{v} f(t)=\Delta^{N} \Delta^{v-N} f(t)
$$

where $t \in \mathbb{N}_{a+v}$ and $0 \leq N-1<v \leq N$.

Lemma 2.3 [3] Let $t$ and $v$ be any numbers for which $t^{(v)}$ and $t^{(v-1)}$ are defined. Then

$$
\Delta t^{(v)}=v t^{(v-1)}
$$

Lemma 2.4 [2] Let $0 \leq N-1<v \leq N$. Then

$$
\Delta^{-v} \Delta^{v} y(t)=y(t)+c_{1} t^{(\nu-1)}+c_{2} t^{(v-2)}+\cdots+c_{N} t^{(\nu-N)}
$$

for some $c_{i} \in R$, with $1 \leq i \leq N$. 
Lemma 2.5 [3] Let $1<v \leq 2$ and $h:[v-1, v+b-1]_{\mathbb{N}_{v-1}} \rightarrow \mathbb{R}$ be given. The unique solution of the FBVP

$$
\begin{aligned}
& -\Delta^{v} y(t)=h(t+v-1), \\
& y(v-2)=0=y(v+b)
\end{aligned}
$$

is given by

$$
y(t)=\sum_{s=0}^{b} G(t, s) h(s+v-1)
$$

where $G:[v-2, v+b]_{\mathbb{N}_{v-2}} \times[0, b]_{\mathbb{N}_{0}} \rightarrow R$ is defined by

$$
G(t, s)=\frac{1}{\Gamma(v)} \begin{cases}\frac{t^{(v-1)}(v+b-s-1)^{(v-1)}}{(v+b)^{(v-1)}}-(t-s-1)^{(v-1)}, & 0 \leq s<t-v+1 \leq b, \\ \frac{t^{(v-1)}(v+b-s-1)^{(v-1)}}{(v+b)(v-1)}, & 0 \leq t-v+1 \leq s \leq b .\end{cases}
$$

Lemma 2.6 [2] The Green's function $G(t, s)$ given in Lemma 2.5 satisfies:

(i) $G(t, s) \geq 0$ for each $(t, s) \in[v-2, v+b]_{\mathbb{N}_{v-2}} \times[0, b]_{\mathbb{N}_{0}}$;

(ii) $\max _{t \in[v-2, v+b]_{\mathbb{N}_{v-2}}} G(t, s)=G(s+v-1, s)$ for each $s \in[0, b]_{\mathbb{N}_{0}}$;

(iii) there exists a number $\gamma \in(0,1)$ such that

$$
\min _{\frac{v+b}{4} \leq t \leq \frac{3(v+b)}{4}} G(t, s) \geq \gamma \max _{t \in[v-2, v+b]_{\mathbb{N}_{v-2}}} G(t, s)=\gamma G(s+v-1, s)
$$

$$
\text { for } s \in[0, b]_{\mathbb{N}_{0}} \text {. }
$$

First of all, we let $\mathcal{B}_{j}$ represent the Banach space of all maps from $\left[v_{j}-2, \ldots, v_{j}+b\right]_{\mathbb{N}_{v_{j}-2}}$ into $\mathbb{R}$ when equipped with the usual maximum norm $\|\cdot\|$. Then, we put $\chi:=\mathcal{B}_{1} \times \mathcal{B}_{2} \times$ $\cdots \times \mathcal{B}_{n}$. By equipping $\chi$ with the norm

$$
\left\|\left(y_{1}, \ldots, y_{n}\right)\right\|=\left\|y_{1}\right\|+\cdots+\left\|y_{n}\right\|,
$$

it follows that $(\chi,\|\cdot\|)$ is a Banach space.

Now consider the operator $S: \chi \rightarrow \chi$ defined by

$$
S\left(y_{1}, \ldots, y_{n}\right)\left(t_{1}, \ldots, t_{n}\right)=\left(S_{1}\left(y_{1}, \ldots, y_{n}\right)\left(t_{1}\right), \ldots, S_{n}\left(y_{1}, \ldots, y_{n}\right)\left(t_{n}\right)\right),
$$

where we define $S_{j}: \chi \rightarrow \mathcal{B}_{j}$ by

$$
\begin{aligned}
S_{j}\left(y_{1}, \ldots, y_{n}\right)\left(t_{j}\right)= & \alpha_{j}\left(t_{j}\right) \psi_{j}\left(y_{j}\right)+\beta_{j}\left(t_{j}\right) \phi_{j}\left(y_{j}\right) \\
& +\lambda_{j} \sum_{s=0}^{b} G_{j}\left(t_{j}, s\right) f_{j}\left(y_{1}\left(s+v_{1}-1\right), \ldots, y_{n}\left(s+v_{n}-1\right)\right),
\end{aligned}
$$

where

$$
\alpha_{j}\left(t_{j}\right):=\frac{1}{\Gamma\left(v_{j}-1\right)}\left[t_{j}^{\left(\nu_{j}-2\right)}-\frac{1}{b+2} t_{j}^{\left(\nu_{j}-1\right)}\right], \quad \beta_{j}\left(t_{j}\right):=\frac{t_{j}^{\left(\nu_{j}-1\right)}}{\left(v_{j}+b\right)^{\left(\nu_{j}-1\right)}} .
$$


Theorem 2.7 Let $f_{j}:[0,+\infty) \times \cdots \times[0,+\infty) \rightarrow[0,+\infty)$ and $\phi_{j}, \psi_{j} \in C\left(\left[v_{j}-2, v_{j}+\right.\right.$ $\left.b]_{\mathbb{N}_{v_{j}-2}}, \mathbb{R}\right)$ be given for $j=1, \ldots, n$, where $C\left(\left[v_{j}-2, v_{j}+b\right]_{\mathbb{N}_{v_{j}-2}}, \mathbb{R}\right)$ stands for the continuous functions on $\left[v_{j}-2, v_{j}+b\right]_{\mathbb{N}_{v_{j}-2}}$. Then $y(t)=\left(y_{1}, \ldots, y_{n}\right) \in \chi$ is a solution of discrete FBVP (1.1)-(1.2) if and only if $y(t)$ is a fixed point of $S$.

Proof From Lemma 2.4, we find that a general solution to problem (1.1)-(1.2) is

$$
y_{j}\left(t_{j}\right)=-\Delta^{-v_{j}} \lambda_{j} f_{j}\left(y_{1}\left(t_{j}+v_{1}-1\right), \ldots, y_{n}\left(t_{j}+v_{n}-1\right)\right)+c_{1 j} t_{j}^{\left(v_{j}-1\right)}+c_{2 j} t_{j}^{\left(v_{j}-2\right)} .
$$

From boundary condition (1.2), we get

$$
\begin{aligned}
y_{j}\left(v_{j}-2\right)= & -\left.\Delta^{-v_{j}} \lambda_{j} f_{j}\left(y_{1}\left(t_{j}+v_{1}-1\right), \ldots, y_{n}\left(t_{j}+v_{n}-1\right)\right)\right|_{t_{j}=v_{j}-2} \\
& +c_{1 j}\left(v_{j}-2\right)^{\left(v_{j}-1\right)}+c_{2 j}\left(v_{j}-2\right)^{\left(v_{j}-2\right)} \\
= & -\left.\frac{1}{\Gamma\left(v_{j}\right)} \sum_{s=0}^{t_{j}-v_{j}}\left(t_{j}-s-1\right)^{\left(v_{j}-1\right)} \lambda_{j} f_{j}\left(y_{1}\left(s+v_{1}-1\right), \ldots, y_{n}\left(s+v_{n}-1\right)\right)\right|_{t_{j}=v_{j}-2} \\
& +c_{2 j} \Gamma\left(v_{j}-1\right) \\
= & c_{2 j} \Gamma\left(v_{j}-1\right)=\psi_{j}\left(y_{j}\right),
\end{aligned}
$$

so

$$
c_{2 j}=\frac{\psi_{j}\left(y_{j}\right)}{\Gamma\left(v_{j}-1\right)} .
$$

On the other hand, applying boundary condition (1.2) to $y_{j}(t)$ implies that

$$
\begin{aligned}
y_{j}\left(v_{j}+b\right)= & -\left.\Delta^{-v_{j}} \lambda_{j} f_{j}\left(y_{1}\left(t_{j}+v_{1}-1\right), \ldots, y_{n}\left(t_{j}+v_{n}-1\right)\right)\right|_{t_{j}=v_{j}+b} \\
& +c_{1 j}\left(v_{j}+b\right)^{\left(v_{j}-1\right)}+c_{2 j}\left(v_{j}+b\right)^{\left(v_{j}-2\right)} \\
= & -\frac{1}{\Gamma\left(v_{j}\right)} \sum_{s=0}^{b}\left(t_{j}-s-1\right)^{\left(v_{j}-1\right)} \lambda_{j} f_{j}\left(y_{1}\left(s+v_{1}-1\right), \ldots, y_{n}\left(s+v_{n}-1\right)\right) \\
& +c_{1 j}\left(v_{j}+b\right)^{\left(v_{j}-1\right)}+\frac{\psi_{j}\left(y_{j}\right)}{\Gamma\left(v_{j}-1\right)}\left(v_{j}+b\right)^{\left(v_{j}-2\right)} \\
= & \phi_{j}\left(y_{j}\right),
\end{aligned}
$$

so

$$
\begin{aligned}
c_{1 j}= & \frac{\phi_{j}\left(y_{j}\right)}{\left(v_{j}+b\right)^{\left(v_{j}-1\right)}}-\frac{\psi_{j}\left(y_{j}\right)\left(v_{j}+b\right)^{\left(v_{j}-2\right)}}{\Gamma\left(v_{j}-1\right)\left(v_{j}+b\right)^{\left(v_{j}-1\right)}} \\
& +\frac{1}{\Gamma\left(v_{j}\right)\left(v_{j}+b\right)^{\left(v_{j}-1\right)}} \sum_{s=0}^{b}\left(v_{j}+b-s-1\right)^{\left(v_{j}-1\right)} \lambda_{j} f_{j}\left(y_{1}\left(s+v_{1}-1\right), \ldots, y_{n}\left(s+v_{n}-1\right)\right) \\
= & \frac{\phi_{j}\left(y_{j}\right)}{\left(v_{j}+b\right)^{\left(v_{j}-1\right)}}-\frac{\psi_{j}\left(y_{j}\right)}{(b+2) \Gamma\left(v_{j}-1\right)} \\
& +\frac{1}{\Gamma\left(v_{j}\right)\left(v_{j}+b\right)^{\left(v_{j}-1\right)}} \sum_{s=0}^{b}\left(v_{j}+b-s-1\right)^{\left(v_{j}-1\right)} \lambda_{j} f_{j}\left(y_{1}\left(s+v_{1}-1\right), \ldots, y_{n}\left(s+v_{n}-1\right)\right) .
\end{aligned}
$$


Finally, we can get that

$$
\begin{aligned}
y_{j}\left(t_{j}\right)= & -\frac{1}{\Gamma\left(v_{j}\right)} \sum_{s=0}^{t_{j}-v_{j}}\left(t_{j}-s-1\right)^{\left(v_{j}-1\right)} \lambda_{j} f_{j}\left(y_{1}\left(s+v_{1}-1\right), \ldots, y_{n}\left(s+v_{n}-1\right)\right) \\
& +\left[\frac{\phi_{j}\left(y_{j}\right)}{\left(v_{j}+b\right)^{\left(v_{j}-1\right)}}-\frac{\psi_{j}\left(y_{j}\right)}{(b+2) \Gamma\left(v_{j}-1\right)}\right] t_{j}^{\left(v_{j}-1\right)} \\
& +\frac{t_{j}^{\left(v_{j}-1\right)}}{\Gamma\left(v_{j}\right)\left(v_{j}+b\right)^{\left(v_{j}-1\right)}} \sum_{s=0}^{b}\left(v_{j}+b-s-1\right)^{\left(v_{j}-1\right)} \lambda_{j} f_{j}\left(y_{1}\left(s+v_{1}-1\right), \ldots, y_{n}\left(s+v_{n}-1\right)\right) \\
& +\frac{\psi_{j}\left(y_{j}\right)}{\Gamma\left(v_{j}-1\right)} t_{j}^{\left(v_{j}-2\right)} \\
= & \psi_{j}\left(y_{j}\right)\left[-\frac{t_{j}^{\left(v_{j}-1\right)}}{(b+2) \Gamma\left(v_{j}-1\right)}+\frac{t_{j}^{\left(v_{j}-2\right)}}{\Gamma\left(v_{j}-1\right)}\right]+\phi_{j}\left(y_{j}\right) \cdot \frac{t_{j}^{\left(v_{j}-1\right)}}{\left(v_{j}+b\right)^{\left(v_{j}-1\right)}} \\
& +\sum_{s=0}^{t_{j}-v_{j}}\left\{\left(\frac{t_{j}^{\left(v_{j}-1\right)}\left(v_{j}+b-s-1\right)^{\left(v_{j}-1\right)}}{\left.\Gamma\left(v_{j}\right)\left(v_{j}+b\right)^{\left(v_{j}-1\right)}-\frac{\left(t_{j}-s-1\right)^{\left(v_{j}-1\right)}}{\Gamma\left(v_{j}\right)}\right)}\right.\right. \\
& \left.\times \lambda_{j} f_{j}\left(y_{1}\left(s+v_{1}-1\right), \ldots, y_{n}\left(s+v_{n}-1\right)\right)\right\} \\
& +\sum_{s=t_{j}-v_{j}+1}^{b} \frac{t_{j}^{\left(v_{j}-1\right)}\left(v_{j}+b-s-1\right)^{\left(v_{j}-1\right)}}{\Gamma\left(v_{j}\right)\left(v_{j}+b\right)^{\left(v_{j}-1\right)}} \lambda_{j} f_{j}\left(y_{1}\left(s+v_{1}-1\right), \ldots, y_{n}\left(s+v_{n}-1\right)\right) \\
= & \psi_{j} \alpha_{j}\left(t_{j}\right)+\phi_{j}\left(y_{j}\right) \beta_{j}\left(t_{j}\right)+\lambda_{j} \sum_{s=0}^{b} G_{j}\left(t_{j}, s\right) f_{j}\left(y_{1}\left(s+v_{1}-1\right), \ldots, y_{n}\left(s+v_{n}-1\right)\right) .
\end{aligned}
$$

The opposite direction is obvious, so it is omitted. Consequently, we get that $y_{j}(t)$ is a solution of (1.1)-(1.2) if and only if $\left(y_{1}, \ldots, y_{n}\right) \in \chi$ is a fixed point of $S$, as desired.

Lemma 2.8 The function $\alpha_{j}\left(t_{j}\right)$ is strictly decreasing in $t_{j}$ for $t_{j} \in\left[v_{j}-2, v_{j}+b\right]_{\mathbb{N}_{v_{j}-2}}$. In addition,

$$
\min _{t_{j} \in\left[v_{j}-2, v_{j}+b\right]_{\mathbb{N}_{v_{j}-2}}} \alpha_{j}\left(t_{j}\right)=0, \quad \max _{t_{j} \in\left[v_{j}-2, v_{j}+b\right]_{\mathbb{N}_{v_{j}-2}}} \alpha_{j}\left(t_{j}\right)=1 .
$$

On the other hand, the function $\beta_{j}\left(t_{j}\right)$ is strictly increasing in $t_{j}$ for $t_{j} \in\left[v_{j}-2, v_{j}+b\right]_{\mathbb{N}_{v_{j}-2}}$. In addition,

$$
\min _{t_{j} \in\left[v_{j}-2, v_{j}+b\right]_{\mathbb{N}_{v_{j}-2}}} \beta_{j}\left(t_{j}\right)=0, \quad \max _{t_{j} \in\left[v_{j}-2, v_{j}+b\right]_{\mathbb{N}_{v_{j}-2}}} \beta_{j}\left(t_{j}\right)=1 .
$$

Proof Note that for every $t_{j} \in\left[v_{j}-2, v_{j}+b\right]_{\mathbb{N}_{v_{j}-2}}$,

$$
\begin{aligned}
\Delta_{t_{j}} \alpha_{j}\left(t_{j}\right) & =\Delta_{t_{j}}\left[\frac{t_{j}^{\left(v_{j}-2\right)}}{\Gamma\left(v_{j}-1\right)}-\frac{t_{j}^{\left(v_{j}-1\right)}}{(b+2) \Gamma\left(v_{j}-1\right)}\right] \\
& =\frac{1}{\Gamma\left(v_{j}-1\right)}\left[\left(v_{j}-2\right) t^{\left(v_{j}-3\right)}-\left(v_{j}-1\right) \frac{t_{j}^{\left(v_{j}-2\right)}}{b+2}\right]<0 .
\end{aligned}
$$


So, the first claim about $\alpha_{j}\left(t_{j}\right)$ holds. On the other hand,

$$
\begin{aligned}
\alpha_{j}\left(v_{j}-2\right) & =\frac{\left(v_{j}-2\right)^{\left(v_{j}-2\right)}}{\Gamma\left(v_{j}-1\right)}-\frac{\left(v_{j}-2\right)^{\left(v_{j}-1\right)}}{(b+2) \Gamma\left(v_{j}-1\right)}=1, \\
\alpha_{j}\left(v_{j}+b\right) & =\frac{\left(v_{j}+b\right)^{\left(v_{j}-2\right)}}{\Gamma\left(v_{j}-1\right)}-\frac{\left(v_{j}+b\right)^{\left(v_{j}-1\right)}}{(b+2)} \Gamma\left(v_{j}-1\right) \\
& =\frac{\Gamma\left(v_{j}+b+1\right)}{\Gamma\left(v_{j}+b+1-v_{j}+2\right) \Gamma\left(v_{j}-1\right)}-\frac{\Gamma\left(v_{j}+b+1\right)}{\Gamma\left(v_{j}+b+1-v_{j}+1\right)(b+2) \Gamma\left(v_{j}-1\right)} \\
& =\frac{\Gamma\left(v_{j}+b+1\right)}{\Gamma(b+3) \Gamma\left(v_{j}-1\right)}-\frac{\Gamma\left(v_{j}+b+1\right)}{\Gamma(b+2)(b+2) \Gamma\left(v_{j}-1\right)} \\
& =0 .
\end{aligned}
$$

It follows that

$$
\max _{t_{j} \in\left[v_{j}-2, v_{j}+b\right]_{\mathbb{N}_{v_{j}-2}}} \alpha_{j}\left(t_{j}\right)=1, \quad \min _{t_{j} \in\left[v_{j}-2, v_{j}+b\right]_{\mathbb{N}_{v_{j}-2}}} \alpha_{j}\left(t_{j}\right)=0 .
$$

It may be shown in a similar way that $\beta_{j}(t)$ satisfies the properties given in the statement of this lemma. We omit the details.

Corollary 1 Let $I_{j}=\left[\frac{b+v_{j}}{4}, \frac{3\left(b+v_{j}\right)}{4}\right]$. There are constants $M_{\alpha_{j}}, M_{\beta_{j}} \in(0,1)$ such that $\min _{t_{j} \in I_{j}} \alpha_{j}\left(t_{j}\right)=$ $M_{\alpha_{j}}\left\|\alpha_{j}\right\|, \min _{t_{j} \in I_{j}} \beta_{j}\left(t_{j}\right)=M_{\beta_{j}}\left\|\beta_{j}\right\|$ for $j=1,2, \ldots, n$, where $\|\cdot\|$ is the usual maximum norm.

Theorem 2.9 [7] Let $\mathcal{B}$ be a Banach space, and let $P \subseteq \mathcal{B}$ be a cone in $\mathcal{B}$. Assume that $S_{1}$ and $S_{2}$ are open subsets of $\mathcal{B}$ with $0 \in S_{1} \subset \bar{S}_{1} \subset S_{2}$. Assume, further, that

$$
T: P \cap\left(\bar{S}_{2} \backslash S_{1}\right) \rightarrow P
$$

is a completely continuous operator. If either

(1) $\|T u\| \leq\|u\|, u \in P \cap \partial S_{1},\|T u\| \geq\|u\|, u \in P \cap \partial S_{2}$; or

(2) $\|T u\| \geq\|u\|, u \in P \cap \partial S_{1},\|T u\| \leq\|u\|, u \in P \cap \partial S_{2}$.

Then $T$ has a fixed point in $P \cap\left(\bar{S}_{2} \backslash S_{1}\right)$.

\section{Main results}

In this section, we present the theorems for the existence of at least two positive solutions to problem (1.1)-(1.2). In the sequel, we let

$$
\begin{aligned}
& A_{j}=\max _{t_{j} \in\left[v_{j}-2, v_{j}+b\right]_{\mathbb{N}_{v_{j}-2}}} \sum_{s=0}^{b} G_{j}\left(t_{j}, s\right), \\
& B_{j}=\min _{t_{j} \in\left[\frac{v_{j}+b}{4}, \frac{3\left(v_{j}+b\right)}{4}\right]} \sum_{s=0}^{b} G_{j}\left(t_{j}, s\right) .
\end{aligned}
$$

We now present the conditions that we presume in the sequel.

$\left(\mathrm{L}_{1}\right) \lim _{\left(y_{1}+\cdots+y_{n}\right) \rightarrow 0^{+}} \frac{f_{i}\left(y_{1}, \ldots, y_{n}\right)}{y_{1}+\cdots+y_{n}}=\infty$ for $t_{j} \in\left[v_{j}-2, v_{j}+b\right]_{\mathbb{N}_{v_{j}-2}}, j=1,2, \ldots, n$. 
(L $\left.\mathrm{L}_{2}\right) \lim _{\left(y_{1}+\cdots+y_{n}\right) \rightarrow \infty} \frac{f_{j}\left(y_{1}, \ldots, y_{n}\right)}{y_{1}+\cdots+y_{n}}=\infty$ for $t_{j} \in\left[v_{j}-2, v_{j}+b\right]_{\mathbb{N}_{v_{j}-2}}, j=1,2, \ldots, n$.

(L $\left.\mathrm{L}_{3}\right) \lim _{\left(y_{1}+\cdots+y_{n}\right) \rightarrow 0^{+}} \frac{f_{j}\left(y_{1}, \ldots, y_{n}\right)}{y_{1}+\cdots+y_{n}}=0$ for $t_{j} \in\left[v_{j}-2, v_{j}+b\right]_{\mathbb{N}_{v^{-2}-2}}, j=1,2, \ldots, n$.

(L $\left.\mathrm{L}_{4}\right) \lim _{\left(y_{1}+\cdots+y_{n}\right) \rightarrow \infty} \frac{f_{j}\left(y_{1}, \ldots, y_{n}\right)}{y_{1}+\cdots+y_{n}}=0$ for $t_{j} \in\left[v_{j}-2, v_{j}+b\right]_{\mathbb{N}_{v_{j}-2}}, j=1,2, \ldots, n$.

$\left(G_{1}\right)$ The functionals $\psi_{j}, \phi_{j}$ are linear. In particular, we assume that

$$
\psi_{j}\left(y_{j}\right)=\sum_{i=v_{j}-2}^{v_{j}+b} c_{i-v_{j}+2}^{j} y_{j}(i), \quad \phi_{j}\left(y_{j}\right)=\sum_{k=v_{j}-2}^{v_{j}+b} d_{k-v_{j}+2}^{j} y_{j}(k)
$$

for $c_{i-v_{j}+2}^{j}, d_{k-v_{j}+2}^{j} \in \mathbb{R}, j=1, \ldots, n$.

$\left(\mathrm{G}_{2}\right)$ For $j=1,2, \ldots, n$, there are

$$
\sum_{i=v_{j}-2}^{v_{j}+b} c_{i-v_{j}+2}^{j} G_{j}(i, s) \geq 0, \quad \sum_{k=v_{j}-2}^{v_{j}+b} d_{k-v_{j}+2}^{j} G_{j}(k, s) \geq 0
$$

for each $s \in[0, b]_{\mathbb{N}_{0}}$, and in addition

$$
\sum_{i=v_{j}-2}^{v_{j}+b} c_{i-v_{j}+2}^{j}+\sum_{k=v_{j}-2}^{v_{j}+b} d_{k-v_{j}+2}^{j} \leq \frac{1}{2 n}
$$

$\left(\mathrm{G}_{3}\right)$ We have that each of $\psi_{j}\left(\alpha_{j}\right), \psi_{j}\left(\beta_{j}\right), \phi_{j}\left(\alpha_{j}\right)$, and $\phi_{j}\left(\beta_{j}\right)$ is nonnegative for $j=1, \ldots, n$.

Let $I:=\left[\frac{v_{1}+b}{4}, \frac{3\left(v_{1}+b\right)}{4}\right] \times \cdots \times\left[\frac{v_{n}+b}{4}, \frac{3\left(v_{n}+b\right)}{4}\right]$. In the sequel, we shall also make use of the cone

$$
\begin{aligned}
\kappa:= & \left\{\left(y_{1}, \ldots, y_{n}\right) \in \chi: y_{1}, \ldots, y_{n} \geq 0, \psi_{j}\left(y_{j}\right) \geq 0, \phi_{j}\left(y_{j}\right) \geq 0, j=1, \ldots, n,\right. \\
& \left.\min _{\left(t_{1}, \ldots, t_{n}\right) \in I}\left[y_{1}\left(t_{1}\right)+\cdots+y_{n}\left(t_{n}\right)\right] \geq \gamma\left\|\left(y_{1}, \ldots, y_{n}\right)\right\|\right\},
\end{aligned}
$$

where $\gamma \in(0,1)$ is a constant defined by

$$
\gamma=\min \left\{\gamma_{1}, \ldots, \gamma_{n}, M_{\alpha_{1}}, \ldots, M_{\alpha_{n}}, M_{\beta_{1}}, \ldots, M_{\beta_{n}}\right\},
$$

where $M_{\alpha_{j}}, M_{\beta_{j}}$ come from Corollary 1 and $\gamma_{j}$ is associated by Lemma 2.6(iii) to $G_{j}\left(t_{j}, s\right)$, $j=1,2, \ldots, n$.

Lemma 3.1 Let $S$ be the operator defined as in (2.2). Then $S: \kappa \rightarrow \kappa$.

Proof By means of $\left(\mathrm{G}_{1}\right)$, we get

$$
\begin{aligned}
\psi_{j}\left(S_{j}\left(y_{1}, \ldots, y_{n}\right)\right) & =\sum_{i=v_{j}-2}^{v_{j}+b} c_{i-v_{j}+2}^{j}\left(S_{j}\left(y_{1}, \ldots, y_{n}\right)\right)(i) \\
& =\sum_{i=v_{j}-2}^{v_{j}+b} \sum_{l=v_{j}-2}^{v_{j}+b} c_{i-v_{j}+2}^{j} c_{l-v_{j}+2}^{j} y_{j}(l) \alpha_{j}(i)
\end{aligned}
$$




$$
\begin{aligned}
& +\sum_{i=v_{j}-2}^{v_{j}+b} \sum_{k=v_{j}-2}^{v_{j}+b} c_{i-v_{j}+2}^{j} d_{k-v_{j}+2}^{j} y_{j}(k) \beta_{j}(i) \\
& +\lambda_{j} \sum_{i=v_{j}-2}^{v_{j}+b} c_{i-v_{j}+2}^{j} \sum_{s=0}^{b} G_{j}(i, s) f_{j}\left(y_{1}\left(s+v_{1}-1\right), \ldots, y_{n}\left(s+v_{n}-1\right)\right) \\
& =\psi_{j}\left(\alpha_{j}\right) \psi_{j}\left(y_{j}\right)+\psi_{j}\left(\beta_{j}\right) \phi_{j}\left(y_{j}\right) \\
& +\lambda_{j} \psi_{j}\left(\sum_{s=0}^{b} G_{j}(t, s) f_{j}\left(y_{1}\left(s+v_{1}-1\right)\right), \ldots, y_{n}\left(s+v_{n}-1\right)\right)
\end{aligned}
$$

for $\left(y_{1}, \ldots, y_{n}\right) \in \chi$.

By assumptions $\left(\mathrm{G}_{2}\right)$ and $\left(\mathrm{G}_{3}\right)$ together with the nonnegativity of $f_{j}\left(y_{1}, \ldots, y_{n}\right)$ and the fact that $\left(y_{1}, \ldots, y_{n}\right) \in \chi$, we can get $\psi_{j}\left(S_{j}\left(y_{1}, \ldots, y_{n}\right)\right) \geq 0$. By means of the same method, we obtain $\phi_{j}\left(S_{j}\left(y_{1}, \ldots, y_{n}\right)\right) \geq 0$ for $j=1,2, \ldots, n$.

On the other hand, we show that

$$
\min _{\left(t_{1}, \ldots, t_{n}\right) \in I}\left[S_{1}\left(y_{1}, \ldots, y_{n}\right)\left(t_{1}\right)+\cdots+S_{n}\left(y_{1}, \ldots, y_{n}\right)\left(t_{n}\right)\right] \geq \gamma\left\|S\left(y_{1}, \ldots, y_{n}\right)\right\|
$$

for $\left(y_{1}, \ldots, y_{n}\right) \in \chi$. In fact, by Lemma 2.6(iii), we have

$$
\begin{aligned}
& \min _{t_{j} \in\left[\frac{b+v_{j}}{4}, \frac{3\left(v_{j}+b\right)}{4}\right]} S_{j}\left(y_{1}, \ldots, y_{n}\right)\left(t_{j}\right) \\
& \geq \min _{t_{j} \in\left[\frac{b+v_{j}}{4}, \frac{3\left(v_{j}+b\right)}{4}\right]} \alpha_{j}\left(t_{j}\right) \psi_{j}\left(y_{j}\right)+\min _{t_{j} \in\left[\frac{b+v_{j}}{4}, \frac{3\left(v_{j}+b\right)}{4}\right]} \beta_{j}\left(t_{j}\right) \phi_{j}\left(y_{j}\right) \\
& +\min _{t_{j} \in\left[\frac{b+v_{j}}{4}, \frac{3\left(v_{j}+b\right)}{4}\right]} \lambda_{j} \sum_{s=0}^{b} G_{j}\left(t_{j}, s\right) f_{j}\left(y_{1}\left(s+v_{1}-1\right), \ldots, y_{n}\left(s+v_{n}-1\right)\right) \\
& \geq M_{\alpha_{j}}\left\|\alpha_{j}\right\| \psi_{j}\left(y_{j}\right)+M_{\beta_{j}}\left\|\beta_{j}\right\| \phi_{j}\left(y_{j}\right) \\
& +\lambda_{j} \sum_{s=0}^{b} \gamma_{j} G_{j}\left(s+v_{j}-1, s\right) f_{j}\left(y_{1}\left(s+v_{1}-1\right), \ldots, y_{n}\left(s+v_{n}-1\right)\right) \\
& \geq \widetilde{\gamma}_{j}\left\|\alpha_{j}\right\| \psi_{j}\left(y_{j}\right)+\widetilde{\gamma}_{j}\left\|\beta_{j}\right\| \phi_{j}\left(y_{j}\right) \\
& +\tilde{\gamma}_{j} \max _{t_{j} \in\left[v_{j}-2, v_{j}+b\right]_{\mathbb{N}_{v_{j}-2}}} \lambda_{j} \sum_{s=0}^{b} G_{j}\left(t_{j}, s\right) f_{j}\left(y_{1}\left(s+v_{1}-1\right), \ldots, y\left(s+v_{n}-1\right)\right) \\
& \geq \tilde{\gamma}_{j} \max _{t_{j} \in\left[v_{j}-2, v_{j}+b\right]_{\mathbb{N}_{v_{j}-2}}}\left[\alpha_{j}\left(t_{j}\right) \psi_{j}\left(y_{j}\right)+\beta_{j} \phi_{j}\left(y_{j}\right)\right. \\
& \left.+\lambda_{j} \sum_{s=0}^{b} G_{j}\left(t_{j}, s\right) f_{j}\left(y_{1}\left(s+v_{1}-1\right), \ldots, y\left(s+v_{n}-1\right)\right)\right] \\
& =\tilde{\gamma}_{j}\left\|S_{j}\left(y_{1}, \ldots, y_{n}\right)\right\| \text {, }
\end{aligned}
$$

where $\tilde{\gamma}_{j}=\left\{M_{\alpha_{j}}, M_{\beta_{j}}, \gamma_{j}\right\}, j=1,2, \ldots, n$. 
Let $\gamma=\min \left\{\gamma_{1}, \ldots, \gamma_{n}, M_{\alpha_{1}}, \ldots, M_{\alpha_{n}}, M_{\beta_{1}}, \ldots, M_{\beta_{n}}\right\}$. Then we obtain

$$
\begin{aligned}
& \min _{\left(t_{1}, \ldots, t_{n}\right) \in I}\left[S_{1}\left(y_{1}, \ldots, y_{n}\right)\left(t_{1}\right)+\cdots+S_{n}\left(y_{1}, \ldots, y_{n}\right)\left(t_{n}\right)\right] \\
& \geq \min _{\left(t_{1}, \ldots, t_{n}\right) \in I} S_{1}\left(y_{1}, \ldots, y_{n}\right)\left(t_{1}\right)+\cdots+\min _{\left(t_{1}, \ldots, t_{n}\right) \in I} S_{n}\left(y_{1}, \ldots, y_{n}\right)\left(t_{n}\right) \\
& \geq \tilde{\gamma}_{1}\left\|S_{1}\left(y_{1}, \ldots, y_{n}\right)\right\|+\cdots+\widetilde{\gamma}_{n}\left\|S_{n}\left(y_{1}, \ldots, y_{n}\right)\right\| \\
& \geq \gamma\left\{\left\|S_{1}\left(y_{1}, \ldots, y_{n}\right)\right\|+\cdots+\left\|S_{n}\left(y_{1}, \ldots, y_{n}\right)\right\|\right\} \\
& =\gamma\left\|S\left(y_{1}, \ldots, y_{n}\right)\right\|
\end{aligned}
$$

for $\left(y_{1}, \ldots, y_{n}\right) \in \chi$.

Finally, by the definitions $S_{j}(j=1,2, \ldots, n)$, it is clear that

$$
S_{j}\left(y_{1}, \ldots, y_{n}\right)\left(t_{j}\right) \geq 0, \quad j=1,2, \ldots n,\left(y_{1}, \ldots, y_{n}\right) \in \chi
$$

So, we conclude that $S: \kappa \rightarrow \kappa$. This completes the proof.

Lemma 3.2 Suppose that conditions $\left(\mathrm{G}_{1}\right)-\left(\mathrm{G}_{3}\right)$ hold, and there exist two different positive numbers $r_{1}, r_{2}, r_{1}<r_{2}$, such that

$$
\begin{aligned}
\max _{0 \leq\left(y_{1}+\cdots+y_{n}\right) \leq r_{1}} f_{j}\left(y_{1}, \ldots, y_{n}\right) \leq & \frac{r_{1}}{2 n \lambda_{j} A_{j}}, \\
\min _{\gamma r_{2} \leq\left(y_{1}+\cdots+y_{n}\right) \leq r_{2}} f_{j}\left(y_{1}, \ldots, y_{n}\right) & \geq \frac{r_{2}}{n \lambda_{j} B_{j}} .
\end{aligned}
$$

Then the operator $S$ has a fixed point $\left(\bar{y}_{1}, \ldots, \bar{y}_{n}\right) \in \kappa$ such that

$$
r_{1} \leq\left\|\left(\bar{y}_{1}, \ldots, \bar{y}_{n}\right)\right\| \leq r_{2}
$$

Proof Let $\kappa_{\xi}=\left\{\left(y_{1}, \ldots, y_{n}\right) \in \kappa,\left\|\left(y_{1}, \ldots, y_{n}\right)\right\|<\xi\right\}$. Then, for any $\left(y_{1}, \ldots, y_{n}\right) \in \kappa$ and $\left\|\left(y_{1}, \ldots, y_{n}\right)\right\|=r_{1}$, we have

$$
\begin{aligned}
\left\|S_{j}\left(y_{1}, \ldots, y_{n}\right)\right\|= & \max _{t_{j} \in\left[v_{j}-2, v_{j}+b\right]_{\mathbb{N}_{v_{j}-2}}} \mid \alpha_{j}\left(t_{j}\right) \psi_{j}\left(y_{j}\right)+\beta_{j}\left(t_{j}\right) \phi_{j}\left(y_{j}\right) \\
& +\lambda_{j} \sum_{s=0}^{b} G_{j}\left(t_{j}, s\right) f_{j}\left(y_{1}\left(s+v_{1}-1\right), \ldots, y_{n}\left(s+v_{n}-1\right)\right) \mid \\
\leq & \sum_{i=v_{j}-2}^{v_{j}+b} c_{i-v_{j}+2}^{j} y_{j}(i)+\sum_{k=v_{j}-2}^{v_{j}+b} d_{k-v_{j}+2}^{j} y_{j}(k) \\
& +\lambda_{j} \max _{t_{j} \in\left[v_{j}-2, v_{j}+b\right]_{\mathbb{N}_{v_{j}-2}}} \sum_{s=0}^{b} G_{j}\left(t_{j}, s\right) f_{j}\left(y_{1}\left(s+v_{1}-1\right), \ldots, y_{n}\left(s+v_{n}-1\right)\right) \\
\leq & r_{1}\left[\sum_{i=v_{j}-2}^{v_{j}+b} c_{i-v_{j}+2}^{j}+\sum_{k=v_{j}-2}^{v_{j}+b} d_{k-v_{j}+2}^{j}\right]+\lambda_{j} A_{j} \frac{r_{1}}{2 n \lambda_{j} A_{j}} \\
\leq & \frac{r_{1}}{2 n}+\frac{r_{1}}{2 n}=\frac{r_{1}}{n}=\frac{1}{n}\left\|\left(y_{1}, \ldots, y_{n}\right)\right\| .
\end{aligned}
$$


That is,

$$
\begin{aligned}
\| S & \left(y_{1}, \ldots, y_{n}\right)\left(t_{1}, \ldots, t_{n}\right) \| \\
& =\left\|\left(S_{1}\left(y_{1}, \ldots, y_{n}\right)\left(t_{1}\right), \ldots, S_{n}\left(y_{1}, \ldots, y_{n}\right)\left(t_{n}\right)\right)\right\| \\
& =\left\|S_{1}\left(y_{1}, \ldots, y_{n}\right)\right\|+\cdots+\left\|S_{n}\left(y_{1}, \ldots, y_{n}\right)\right\| \\
& \leq \frac{1}{n}\left\|\left(y_{1}, \ldots, y_{n}\right)\right\|+\cdots+\frac{1}{n}\left\|\left(y_{1}, \ldots, y_{n}\right)\right\| \\
& =\left\|\left(y_{1}, \ldots, y_{n}\right)\right\|
\end{aligned}
$$

for $\left(y_{1}, \ldots, y_{n}\right) \in \partial \kappa_{r_{1}}$.

On the other hand, for any $\left(y_{1}, \ldots, y_{n}\right) \in \kappa$ and $\left\|\left(y_{1}, \ldots, y_{n}\right)\right\|=r_{2}$, we have

$$
\begin{aligned}
\left\|S_{j}\left(y_{1}, \ldots, y_{n}\right)\right\| \geq & \alpha_{j}\left(t_{j}\right) \psi_{j}\left(y_{j}\right)+\beta_{j}\left(t_{j}\right) \phi_{j}\left(y_{j}\right) \\
& +\lambda_{j} \sum_{s=0}^{b} G_{j}\left(t_{j}, s\right) f_{j}\left(y_{1}\left(s+v_{1}-1\right), \ldots, y_{n}\left(s+v_{n}-1\right)\right) \\
\geq & \lambda_{j} \sum_{s=0}^{b} G_{j}\left(t_{j}, s\right) f_{j}\left(y_{1}\left(s+v_{1}-1\right), \ldots, y_{n}\left(s+v_{n}-1\right)\right) \\
\geq & \min _{t_{j} \in\left[\frac{v_{j}+b}{4}, \frac{3\left(v_{j}+b\right)}{4}\right]} \lambda_{j} \sum_{s=0}^{b} G_{j}\left(t_{j}, s\right) f_{j}\left(y_{1}\left(s+v_{1}-1\right), \ldots, y_{n}\left(s+v_{n}-1\right)\right) \\
\geq & \min _{t_{j} \in\left[\frac{v_{j}+b}{4}, \frac{3\left(v_{j}+b\right)}{4}\right]} \lambda_{j} \sum_{s=0}^{b} G_{j}\left(t_{j}, s\right) \frac{r_{2}}{n \lambda_{j} B_{j}} \\
= & \frac{r_{2}}{n}=\frac{1}{n}\left\|\left(y_{1}, \ldots, y_{n}\right)\right\| .
\end{aligned}
$$

That is,

$$
\begin{aligned}
\| S & \left(y_{1}, \ldots, y_{n}\right)\left(t_{1}, \ldots, t_{n}\right) \| \\
& =\left\|\left(S_{1}\left(y_{1}, \ldots, y_{n}\right)\left(t_{1}\right), \ldots, S_{n}\left(y_{1}, \ldots, y_{n}\right)\left(t_{n}\right)\right)\right\| \\
& =\left\|S_{1}\left(y_{1}, \ldots, y_{n}\right)\right\|+\cdots+\left\|S_{n}\left(y_{1}, \ldots, y_{n}\right)\right\| \\
& \geq \frac{1}{n}\left\|\left(y_{1}, \ldots, y_{n}\right)\right\|+\cdots+\frac{1}{n}\left\|\left(y_{1}, \ldots, y_{n}\right)\right\| \\
& =\left\|\left(y_{1}, \ldots, y_{n}\right)\right\|
\end{aligned}
$$

for $\left(y_{1}, \ldots, y_{n}\right) \in \partial \kappa_{r_{2}}$.

By the use of Theorem 2.9, there exists $\left(\bar{y}_{1}, \ldots, \bar{y}_{n}\right) \in \kappa$ such that $S\left(\bar{y}_{1}, \ldots, \bar{y}_{n}\right)=\left(\bar{y}_{1}, \ldots, \bar{y}_{n}\right)$, the proof is complete.

Theorem 3.3 Suppose that conditions $\left(\mathrm{L}_{1}\right),\left(\mathrm{L}_{2}\right)$ and $\left(\mathrm{G}_{1}\right)-\left(\mathrm{G}_{3}\right)$ hold. Then, for every $\lambda_{j} \in$ $\left(0, \lambda_{j}^{*}\right)$, problem (1.1)-(1.2) has at least two positive solutions, where

$$
\lambda_{j}^{*}=\frac{1}{2 n A_{j}} \sup _{r>0} \frac{r}{\max _{0 \leq y_{1}+\cdots+y_{n} \leq r} f_{j}\left(y_{1}, \ldots, y_{n}\right)} .
$$


Proof Define the function

$$
p_{j}(r)=\frac{r}{2 n A_{j} \max _{0 \leq y_{1}+\cdots+y_{n} \leq r} f_{j}\left(y_{1}, \ldots, y_{n}\right)}, \quad j=1, \ldots, n,
$$

we have that $p_{j} \in C((0, \infty),(0, \infty))$. In view of $\left(\mathrm{L}_{1}\right)$, we see that $\lim _{r \rightarrow 0} \frac{r}{f_{j}(r)}=0$, that is, $\lim _{r \rightarrow 0} \frac{r}{2 n A_{j} f_{j}(r)}=0$, and

$$
0<p_{j}(r)=\frac{r}{2 n A_{j} \max _{0 \leq y_{1}+\cdots+y_{n} \leq r} f_{j}\left(y_{1}, \ldots, y_{n}\right)} \leq \frac{r}{2 n A_{j} f_{j}(r)},
$$

so $\lim _{r \rightarrow 0} p_{j}(r)=0$.

In view of $\left(\mathrm{L}_{2}\right)$, we see further that $\lim _{r \rightarrow \infty} p_{j}(r)=0$. Thus, there exists $r_{0}>0$ such that $p_{j}\left(r_{0}\right)=\max _{r>0} p_{j}(r)=\lambda_{j}^{*}, j=1, \ldots, n$. For any $\lambda_{j} \in\left(0, \lambda_{j}^{*}\right)$, by the intermediate value theorem, there exist two points $b_{1} \in\left(0, r_{0}\right), b_{2} \in\left(r_{0}, \infty\right)$ such that $p_{j}\left(b_{1}\right)=p_{j}\left(b_{2}\right)=\lambda_{j}$. Thus, we have

$$
\begin{array}{ll}
f_{j}\left(y_{1}, \ldots, y_{n}\right) \leq \frac{b_{1}}{2 n \lambda_{j} A_{j}}, \quad y_{1}+\cdots+y_{n} \in\left[0, b_{1}\right] \\
f_{j}\left(y_{1}, \ldots, y_{n}\right) \leq \frac{b_{2}}{2 n \lambda_{j} A_{j}}, \quad y_{1}+\cdots+y_{n} \in\left[0, b_{2}\right] .
\end{array}
$$

On the other hand, in view of $\left(\mathrm{L}_{1}\right)$ and $\left(\mathrm{L}_{2}\right)$, we see that there exist $c_{1} \in\left(0, b_{1}\right), c_{2} \in\left(b_{2}, \infty\right)$ such that

$$
\frac{f_{j}\left(y_{1}, \ldots, y_{n}\right)}{y_{1}+\cdots+y_{n}} \geq \frac{1}{n \lambda_{j} \gamma B_{j}}, \quad y_{1}+\cdots+y_{n} \in\left(0, b_{1}\right] \cup\left[b_{2} \gamma, \infty\right)
$$

That is,

$$
\begin{array}{ll}
f_{j}\left(y_{1}, \ldots, y_{n}\right) \geq \frac{c_{1}}{n \lambda_{j} B_{j}}, & y_{1}+\cdots+y_{n} \in\left[\gamma c_{1}, c_{1}\right], \\
f_{j}\left(y_{1}, \ldots, y_{n}\right) \geq \frac{c_{2}}{n \lambda_{j} B_{j}}, & y_{1}+\cdots+y_{n} \in\left[\gamma c_{2}, c_{2}\right],
\end{array}
$$

where $\gamma$ is defined by (3.5). An application of Lemma 3.2 leads to two distinct solutions of (1.1)-(1.2) which satisfy

$$
c_{1} \leq\left\|\left(\bar{y}_{1}, \ldots, \bar{y}_{n}\right)\right\| \leq b_{1}, \quad b_{2} \leq\left\|\left(\bar{y}_{1}^{\prime}, \ldots, \bar{y}_{n}^{\prime}\right)\right\| \leq c_{2} .
$$

The proof is complete.

Theorem 3.4 Suppose that $\left(\mathrm{L}_{3}\right),\left(\mathrm{L}_{4}\right)$ and $\left(\mathrm{G}_{1}\right)-\left(\mathrm{G}_{3}\right)$ hold. Then, for any $\lambda_{j} \geq \lambda_{j}^{* *}$, equation (1.1)-(1.2) has at least two positive solutions, where

$$
\lambda_{j}^{* *}=\frac{1}{n B_{j}} \inf _{r>0} \frac{r}{\min _{\gamma r \leq y_{1}+\cdots+y_{n} \leq r} f_{j}\left(y_{1}, \ldots, y_{n}\right)},
$$

and $\gamma$ is defined by (3.5). 
The proof is similar to Theorem 3.3 and hence omitted.

We now present an example to illustrate the sorts of boundary conditions that can be treated by Theorem 3.3 .

Example 3.1 Consider the following boundary value problems:

$$
\begin{aligned}
& \left\{\begin{array}{l}
\Delta^{\frac{13}{10}} y(t)=-\lambda_{1} f_{1}\left(y_{1}\left(t+\frac{3}{10}\right), y_{2}\left(t+\frac{1}{2}\right)\right), \\
\Delta^{\frac{3}{2}} y(t)=-\lambda_{2} f_{2}\left(y_{1}\left(t+\frac{3}{10}\right), y_{2}\left(t+\frac{1}{2}\right)\right),
\end{array}\right. \\
& y_{1}\left(-\frac{7}{10}\right)=\frac{1}{12} y_{1}\left(\frac{13}{10}\right)-\frac{1}{25} y_{1}\left(\frac{53}{10}\right), \\
& y_{1}\left(\frac{213}{10}\right)=\frac{1}{30} y_{1}\left(\frac{83}{10}\right)-\frac{1}{100} y_{1}\left(\frac{73}{10}\right), \\
& y_{2}\left(-\frac{1}{2}\right)=\frac{1}{40} y_{2}\left(\frac{3}{2}\right)-\frac{1}{150} y_{2}\left(\frac{15}{2}\right), \\
& y_{2}\left(\frac{43}{2}\right)=\frac{1}{17} y_{2}\left(\frac{5}{2}\right)-\frac{1}{30} y_{2}\left(\frac{11}{2}\right),
\end{aligned}
$$

where $b=20, v_{1}=\frac{13}{10}, v_{2}=\frac{3}{2}$, we take

$$
\begin{array}{ll}
f_{1}\left(y_{1}, y_{2}\right)=\left(y_{1}+y_{2}\right)^{\frac{1}{2}}+\left(y_{1}+y_{2}\right)^{2}, & f_{2}\left(y_{1}, y_{2}\right)=\left(y_{1}+y_{2}\right)^{\frac{1}{2}}+\frac{1}{64}\left(y_{1}+y_{2}\right)^{\frac{3}{2}}, \\
\psi_{1}\left(y_{1}\right)=\frac{1}{12} y_{1}\left(\frac{13}{10}\right)-\frac{1}{25} y_{1}\left(\frac{53}{10}\right), & \phi_{1}\left(y_{1}\right)=\frac{1}{30} y_{1}\left(\frac{83}{10}\right)-\frac{1}{100} y_{1}\left(\frac{73}{10}\right), \\
\psi_{2}\left(y_{2}\right)=\frac{1}{40} y_{2}\left(\frac{3}{2}\right)-\frac{1}{150} y_{2}\left(\frac{15}{2}\right), & \phi_{2}\left(y_{2}\right)=\frac{1}{17} y_{2}\left(\frac{5}{2}\right)-\frac{1}{30} y_{2}\left(\frac{11}{2}\right),
\end{array}
$$

$f_{1}, f_{2}:[0,+\infty) \times[0,+\infty) \rightarrow[0,+\infty)$, and $y_{1}$ is defined on the time scale $\left\{-\frac{7}{10}, \frac{3}{10}, \ldots, \frac{213}{10}\right\}$, $y_{2}$ is defined on the time scale $\left\{-\frac{1}{2}, \frac{1}{2}, \ldots, \frac{43}{2}\right\}$.

It is easy to get that $\left(\mathrm{F}_{1}\right),\left(\mathrm{F}_{2}\right)$ hold. On the other hand, $\left(\mathrm{G}_{1}\right)$ holds. Now, we see that $\left(\mathrm{G}_{2}\right)$, $\left(\mathrm{G}_{3}\right)$ hold. In fact,

$$
\begin{aligned}
& \sum_{i=\nu_{1}-2}^{\nu_{1}+b} c_{i-\nu_{1}+2}^{1}+\sum_{k=\nu_{1}-2}^{\nu_{1}+b} d_{k-\nu_{1}+2}^{1}=\frac{1}{12}-\frac{1}{25}+\frac{1}{30}-\frac{1}{100}=\frac{1}{15}<\frac{1}{4}, \\
& \sum_{i=\nu_{2}-2}^{\nu_{2}+b} c_{i-\nu_{2}+2}^{2}+\sum_{k=\nu_{2}-2}^{\nu_{2}+b} d_{k-\nu_{2}+2}^{2}=\frac{1}{40}-\frac{1}{150}+\frac{1}{17}-\frac{1}{30}=\frac{149}{3,400}<\frac{1}{4} .
\end{aligned}
$$

In addition,

$$
\begin{aligned}
& \sum_{i=\nu_{1}-2}^{v_{1}+b} c_{i-v_{1}+2}^{1} G_{1}(i, s) \\
& \quad=\frac{1}{12} G_{1}\left(v_{1}, s\right)-\frac{1}{25} G_{1}\left(v_{1}+4, s\right) \\
& \quad=\frac{1}{12} \frac{v_{1}^{\left(\nu_{1}-1\right)}\left(v_{1}+b-s-1\right)^{\left(\nu_{1}-1\right)}}{\Gamma\left(v_{1}\right)\left(v_{1}+b\right)^{\left(v_{1}-1\right)}}-\frac{1}{25} G\left(v_{1}+4, s\right)
\end{aligned}
$$




$$
\begin{aligned}
& \geq \frac{1}{12} \frac{v_{1}^{\left(v_{1}-1\right)}\left(v_{1}+b-s-1\right)^{\left(v_{1}-1\right)}}{\Gamma\left(v_{1}\right)\left(v_{1}+b\right)^{\left(v_{1}-1\right)}}-\frac{1}{25} \frac{\left(v_{1}+4\right)^{\left(v_{1}-1\right)}\left(v_{1}+b-s-1\right)^{\left(v_{1}-1\right)}}{\Gamma\left(v_{1}\right)\left(v_{1}+b\right)^{\left(\nu_{1}-1\right)}} \\
& =\frac{\left(v_{1}+b-s-1\right)^{\left(v_{1}-1\right)}}{\Gamma\left(v_{1}\right)\left(v_{1}+b\right)^{\left(\nu_{1}-1\right)}}\left[\frac{1}{12} v_{1}^{\left(\nu_{1}-1\right)}-\frac{1}{25}\left(v_{1}+4\right)^{\left(\nu_{1}-1\right)}\right] \\
& =\frac{v_{1}\left(v_{1}+b-s-1\right)^{\left(\nu_{1}-1\right)}}{\left(v_{1}+b\right)^{\left(\nu_{1}-1\right)}} \cdot \frac{16}{625}>0 \text {. }
\end{aligned}
$$

By using a similar method, we get

$$
\sum_{k=\nu_{1}-2}^{\nu_{1}+b} d_{k-\nu_{1}+2}^{1} G_{1}(k, s) \geq 0, \quad \sum_{i=\nu_{2}-2}^{\nu_{2}+b} c_{i-\nu_{2}+2}^{2} G_{2}(i, s) \geq 0, \quad \sum_{k=\nu_{2}-2}^{\nu_{2}+b} d_{k-\nu_{2}+2}^{2} G_{2}(k, s) \geq 0 .
$$

Hence, $\left(G_{2}\right)$ holds.

Finally, we numerically calculate that

$$
\begin{aligned}
\psi_{1}\left(\alpha_{1}\right)= & \frac{1}{12} \alpha_{1}\left(v_{1}\right)-\frac{1}{25} \alpha_{1}\left(v_{1}+4\right) \\
= & \frac{1}{12 \Gamma\left(v_{1}-1\right)}\left[v_{1}^{\left(v_{1}-2\right)}-\frac{1}{b+2} v_{1}^{\left(v_{1}-1\right)}\right] \\
& -\frac{1}{25 \Gamma\left(v_{1}-1\right)}\left[\left(v_{1}+4\right)^{\left(v_{1}-2\right)}-\frac{1}{b+2}\left(v_{1}+4\right)^{\left(v_{1}-1\right)}\right] \\
\approx & 0.012 .
\end{aligned}
$$

Similarly, we have

$$
\begin{aligned}
& \psi_{1}\left(\beta_{1}\right) \approx 0.012, \quad \phi_{1}\left(\alpha_{1}\right) \approx 0.00091, \quad \phi_{1}\left(\beta_{1}\right) \approx 0.018 \\
& \psi_{2}\left(\alpha_{2}\right) \approx 0.00769, \quad \psi_{2}\left(\beta_{2}\right) \approx 0.00315, \quad \phi_{2}\left(\alpha_{2}\right) \approx 0.0104, \\
& \phi_{2}\left(\beta_{2}\right) \approx 0.0038 .
\end{aligned}
$$

We obtain that each of $\psi_{j}\left(\alpha_{j}\right), \psi_{j}\left(\beta_{j}\right), \phi_{j}\left(\alpha_{j}\right)$ and $\phi_{j}\left(\beta_{j}\right)$ is nonnegative for $j=1,2$. So, condition $\left(\mathrm{G}_{3}\right)$ holds. Namely, $f_{1}, f_{2}$ and $\psi_{1}, \psi_{2}, \phi_{1}, \phi_{2}$ satisfy the conditions of Theorem 3.3. A computation shows that $\lambda_{1}^{*} \approx 5.33 \times 10^{-3}, \lambda_{2}^{*} \approx 1.357 \times 10^{-2}$. Then, for every $\lambda_{j} \in$ $\left(0, \lambda_{j}^{*}\right)(j=1,2)$, problem (3.10)-(3.12) has at least two positive solutions.

Competing interests

The authors declare that they have no competing interests.

\section{Authors' contributions}

SK conceived of the study, and participated in its design and coordination. XZ drafted the manuscript. HC participated in the design of the study and the sequence correction. All authors read and approved the final manuscript.

\section{Author details}

${ }^{1}$ Applied of Mathematics Institute, Shanxi Datong University, Datong, Shanxi 037009, P.R. China. ${ }^{2}$ Department of Mathematics, Shanxi Normal University, Linfen, Shanxi 041000, P.R. China. 


\section{Acknowledgements}

The authors are very grateful to the reviewers for their valuable suggestions and useful comments, which led to an improvement of this paper. Project supported by the National Natural Science Foundation of China (Grant No. 11271235) and Shanxi Province (2008011002-1) and Shanxi Datong University Institute (2009-Y-15, 2010-B-01, 2013K5) and the Development Foundation of Higher Education Department of Shanxi Province (20101109, 20111020).

Received: 19 June 2013 Accepted: 5 December 2013 Published: 20 Dec 2013

\section{References}

1. Henderson, J, Ntouyas, SK, Purnaras, IK: Positive solutions for systems of nonlinear discrete boundary value problems. Differ. Equ. Appl. 15, 895-912 (2009)

2. Goodrich, CS: Existence of a positive solution to a system of discrete fractional boundary value problems. Comput. Math. Appl. 217, 4740-4753 (2011)

3. Atici, FM, Eloe, PW: Fractional q-calculus on a time scale. J. Nonlinear Math. Phys. 14(3), 333-344 (2007)

4. Atici, FM, Eloe, PW: A transform method in discrete fractional calculus. Int. J. Differ. Equ. 2(2), 165-176 (2007)

5. Atici, FM, Eloe, PW: Initial value problems in discrete fractional calculus. Proc. Am. Math. Soc. 137(3), 981-989 (2009)

6. Atici, FM, Eloe, PW: Two-point boundary value problems for finite fractional difference equations. J. Differ. Equ. Appl. 17(4), 445-456 (2011)

7. Ge, W: Boundary Value Problems for Nonlinear Ordinary Differential Equations. Science Press, Beijing (2007)

10.1186/1687-1847-2013-376

Cite this article as: Kang et al.: Positive solutions for boundary value problems of fractional difference equations depending on parameters. Advances in Difference Equations 2013, 2013:376

\section{Submit your manuscript to a SpringerOpen ${ }^{\circ}$ journal and benefit from:}

- Convenient online submission

- Rigorous peer review

Immediate publication on acceptance

Open access: articles freely available online

High visibility within the field

Retaining the copyright to your article 